${ }^{1}$ Departamento de Economia, Centro de Ciências Sociais Aplicadas, Universidade Federal da Paraíba. Cidade Universitária, Castelo Branco. 58059-900 João Pessoa PB Brasil. julianadantas07@gmail.com

\title{
Uma análise da prevenção do câncer de mama no Brasil
}

\author{
An analysis of breast cancer prevention in Brazil
}

Abstract This research sets out to study the interrelationship between breast cancer prevention and the socioeconomic, demographic, behavioral, regional and health factors in determining the temporal frequency of tests for prevention by performing mammograms and breast exams in Brazil. Based on the information of the health supplement of the National Sampling Survey of Domiciles of 2008, a sample that includes only women over age 40 was created. To achieve the desired goal, the estimate of the ordered logit model is used to establish partial proportional odds, which revealed the main results as follows. Women with a good socioeconomic status, living in the more developed regions of the country, family structure including children, making a good self-assessment of health and having been diagnosed with some type of cancer in the past. This is the profile of women who are best at preventing breast cancer, both in relation to the demand for mammograms and to a greater demand for breast exam performed by a doctor or a nurse. The results also point to the fact that the majority of those interviewed had either had the tests in question within the previous year, or had never been exposed to such procedures.

Key words Breast cancer, Prevention, Economic aspects, Ordered logit
Resumo A presente pesquisa se propõe a estudar as inter-relações entre a prevenção do câncer de mama e os fatores socioeconômicos, demográficos, comportamentais, regionais e de saúde na determinação da frequência temporal à busca por prevenção via realização de mamografias e exames de mama no Brasil. A partir das informações do suplemento de saúde da Pesquisa Nacional por Amostra de Domicílios, de 2008, foi construída uma amostra que contempla apenas mulheres com mais de 40 anos de idade. Para o atendimento do objetivo proposto, faz-se uso da estimação do modelo logit, ordenado de chances proporcionais parciais, que indicaram como principais resultados: i) melhores condições socioeconômicas, residir nas regiões mais desenvolvidas do país, uma composição familiar que inclua filhos, apresentar uma boa autoavaliação da própria saúde e já ter sido diagnosticada com algum tipo de câncer, desenham o perfil da mulher que mais se previne contra o câncer de mama, tanto em relação à procura por mamografia, como a uma maior demanda por exame de mama realizado por médico ou enfermeiro e; ii) os resultados apontam ainda para o fato de que grande parte das entrevistadas realizaram os exames em questão há pelo menos um ano, ou nunca passaram por tais procedimentos.

Palavras chave Câncer de mama, Prevenção, Aspectos econômicos, Logit ordenado 


\section{Introdução}

A incidência do câncer de mama constitui-se uma pandemia global, atingindo tanto países desenvolvidos como nações em desenvolvimento. Chama a atenção que a prevalência de tumor maligno mamário (em mulheres) é mais recorrente nos países desenvolvidos. No entanto, as mulheres diagnosticadas com câncer de mama nas nações mais ricas, conseguem vistoria clínica precoce e tratamentos mais precisos, vis-à-vis às mulheres dos países em desenvolvimento. Isto é, a relação incidência-mortalidade é menor nos países desenvolvidos ${ }^{1,2}$.

Por definição, câncer é o nome dado a um conjunto de mais de 100 doenças que têm em comum o crescimento desordenado (maligno) de células que invadem os tecidos e órgãos, podendo espalhar-se (metástase) para outras regiões do corpo ${ }^{3}$.

Especificamente, o câncer de mama é um tumor maligno que se desenvolve no tecido da mama. Essa neoplasia é um problema que está despertando maior atenção na saúde pública mundial, nesse contexto, no ano de 2012 foram registrados cerca de 1,67 milhões de novos casos em todo o mundo ${ }^{1}$. No Brasil não é diferente, esse tipo de câncer é o mais comum entre as mulheres e o segundo mais frequente ${ }^{1}$. Nesse contexto, é fundamental alertar e orientar a população feminina sobre a importância da prevenção, pois os tumores mamários malignos estão entre as principais causas de óbito no Brasil.

O diagnóstico em estágios avançados da doença reduz as chances de cura e é um dos fatores responsáveis pela alta taxa de mortalidade. Isto posto, o acesso limitado da população ao tratamento, seja devido à distribuição desigual da renda ou escassez de atendimento do serviço público, leva ao aumento do número de óbitos registrados.

As ações de prevenção ajudam a minimizar o custo de cuidado com saúde, além de melhorar a qualidade de vida das pessoas. As consequências de uma doença crônica podem atingir os aspectos sociais e econômicos do país, dificultando seu desenvolvimento. $\mathrm{O}$ tratamento com a doença, a perda de produtividade e os custos com a saúde são despesas sociais e econômicas para os indivíduos $^{4-7}$.

Vale destacar que as mudanças no estilo de vida das mulheres tendem a aumentar os fatores de risco da doença, associado a ocorrências tais como: ausência da maternidade, realização de intervenção hormonal, a maternidade pós 30 anos de idade, bem como maus hábitos como: seden- tarismo, má alimentação, obesidade, tabagismo e consumo de álcool em excesso, além do histórico familiar de câncer, sendo a idade o principal fator de risco para o diagnóstico do câncer de mama, no qual a faixa etária de incidência é mais frequente em mulheres acima dos 40 anos. Assim, apesar dos avanços com programas e propaganda de prevenção, o número de brasileiras diagnosticadas com a doença continua a se elevar. Até o fim de 2014, no Brasil, são esperados 57.120 novos casos do câncer de mama, ou seja, são 56,09 casos a cada 100 mil mulheres ${ }^{1}$.

Segundo Ramirez et al. ${ }^{8}$ e Wojcik et al. ${ }^{9}$, os riscos com o câncer de mama são maiores entre as mulheres afro-americanas, as razões para as diferenças de raça e etnia não são claras, mas muitos estudos epidemiológicos sugerem que essa desigualdade é em grande parte devido a resultado de fatores socioeconômicos como pobreza, que proporciona um acesso desigual à qualidade de saúde, reduzindo as taxas de acesso à mamografia, além de outros fatores socioeconômicos em conjunto, como o comportamental e o cultural, explicam melhor as fases do momento do diagnóstico e da sobrevivência. É importante destacar que a presença de câncer nas mulheres não pode ser exatamente evitada, pois depende também de fatores genéticos que fogem do controle da mulher em sua totalidade.

Portanto, é importante estudar na ciência econômica, a prevenção do câncer de mama, haja vista que medidas preventivas ajudam a minimizar o custo de cuidado com saúde. Empresas, famílias e a sociedade como um todo são afetadas pelos prejuízos causados pelo diagnóstico da doença aqui tratada. Entre os custos, é possível mencionar perda de produtividade no trabalho, consequências emocionais, aumento das despesas de saúde com gastos médicos, redução da expectativa de vida e mortalidade precoce. Assim, as políticas nacionais de saúde necessitam de aprimoramento para melhorar a qualidade de vida da população feminina ${ }^{7}$.

No Brasil, alguns esforços já foram realizados com o intuito de entender os determinantes da demanda por prevenção ao câncer de mama para mulheres de 25 anos ou mais. Oliveira et al. ${ }^{10}$ estimaram as chances de as mulheres realizarem mamografia no Brasil, nos anos de 2003 e 2008, fazendo uso de um logit binário, com o qual chegaram à conclusão de que as de maior renda e escolaridade, bem como as casadas e as que têm plano de saúde são as que apresentam a maior chance de procurarem a realização de uma mamografia. 
Já Matos et al. ${ }^{11}$ investigaram os determinantes associados a prevenção - prevenção definida como a realização de autoexame, exame clínico nas mamas e mamografia - das mulheres do Município de Maringá/PR, em uma pesquisa de campo realizada no ano de 2008. Os referidos autores tomaram como público mulheres de 40 a 69 anos de idade, e seus resultados apontam que a maior busca por prevenção está também associada a melhores status socioeconômicos, além do destaque dado à realização de tratamento para a menopausa e aos aspectos religiosos. De conformidade com os resultados anteriormente mencionados, Oliveira e Kretzmann ${ }^{12}$ concluíram que os principais determinantes para a demanda por prevenção do câncer de mama são a idade, a escolaridade e o nível de renda.

Portanto, diante do exposto, a presente pesquisa objetiva é apresentar a inter-relação entre a prevenção do câncer de mama e fatores socioeconômicos, demográficos, comportamentais, regionais e de saúde, para o grupo de mulheres com mais de 40 anos de idade. Assim, este artigo apresenta uma contribuição à literatura brasileira, pois avança em algumas questões, quando se compara a outros trabalhos, pois modela as variáveis dependentes em mais de 2 categorias e considera a configuração do plano amostral complexo da PNAD. Oliveira et al. ${ }^{10}$ e Oliveira e Kretzmann ${ }^{12}$ categorizam o exame de mama e mamografia em apenas 2 categorias, dado que utilizam o modelo binário, além de não considerar o efeito do plano amostral da base de dados. Já Matos et al. ${ }^{11}$ restringem sua análise apenas para o município de Maringá-PR.

\section{Métodos}

\section{Base de dados: fonte e tratamento e descrição das variáveis}

Para aferir sobre a demanda por prevenção do câncer de mama das mulheres brasileiras, utilizou-se como indicadores de prevenção a quantidade de exames de toque nas mamas ou mamografias realizadas ao longo do tempo, subdivididas, entre as que realizaram os procedimentos há pelo menos um ano e as que nunca o realizaram. A base de dados utilizada no trabalho foi o suplemento de saúde da (PNAD) 2008.

Como a PNAD é uma pesquisa que possui desenho amostral complexo, ou seja, utiliza amostragem estratificada e de conglomerados e ainda com probabilidades de seleção desiguais, todas as estatísticas e modelos econométricos utilizados neste trabalho consideraram o efeito do desenho do plano amostral em suas estimativas.

O conjunto de dados da PNAD, para o ano de 2008, apresentava inicialmente 391.868 pessoas, retirados os homens restaram 201.288 entrevistadas, outro recorte fora feito, mantendo na amostra apenas as mulheres com mais de 40 anos de idade. Entre as razões para conservarmos na amostra apenas mulheres com esta faixa etária, destacam-se: i) relativa baixa incidência de câncer de mama em mulheres com menos de 40 anos; ii) a indicação da OMS de que as mulheres devem realizar mamografia a partir dos 40 anos; iii) mulheres com mais de 40 anos são mais propensas a desenvolverem tumores mamários malignos e; iv) indicação da revisão de literatura ${ }^{13,14}$. Sendo assim, após o ajustamento nos dados, a amostra contempla um total de 67.511 entrevistadas.

Com base nas informações anteriormente apresentadas, que se pautou pela descrição das variáveis, bem como sua fonte e tratamento, o próximo passo da pesquisa consistiu em apresentar algumas características descritivas da amostra, reportadas nas Tabelas 1 e 2. A Tabela 1 mostra a distribuição de frequência e o percentual em relação às medidas preventivas da população feminina entrevistada, tanto em relação a mamografia quanto relacionadas ao exame das mamas.

Referente à mamografia foi indagado às entrevistadas: "Quando foi a última vez que fez a mamografia? " Onde as respostas foram categorizadas em: 1) se realizou o exame até um ano; 2) para mais de até dois anos; 3 ) se fazia mais de dois anos e; 4) nunca fez o exame.

Por outro lado, ao serem perguntadas quando foi a última vez que tinham feito exame clínico das mamas, executado por médico ou enfermeiro, os retornos foram: 1) se realizou o exame até um ano; 2) se para mais de um até dois anos; 3 ) acima de dois anos e; 4) nunca fez.

A partir da Tabela 1, é possível perceber que a maioria das mulheres entrevistadas havia feito a mamografia há pelo menos um ano ou então nunca tinham feito o procedimento, representando $35,00 \%$ e $32,96 \%$, respectivamente. Por outro lado, referente ao exame das mamas, é possível perceber que 39,66\% o tinham realizado há pelo menos um ano, e 25,25\% nunca o fizeram. Ou seja, independente de considerar-se o exame de toque nas mamas, realizado por profissional de saúde, ou o exame de mamografia computadorizado, é possível perceber que grande parte da amostra encontra-se nos extremos, indicando que realizaram o exame a menos de um ano 


\begin{tabular}{|c|c|c|c|}
\hline 3166 & & & \\
\hline 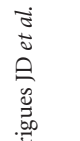 & & & \\
\hline$\stackrel{\square}{\mathscr{C}}$ & $\begin{array}{l}\text { Tabela 1. Frequência da mam } \\
\text { mama. }\end{array}$ & mografia e do & xame de \\
\hline & & Mamografia & $\begin{array}{c}\text { Exame da } \\
\text { mama }\end{array}$ \\
\hline & & Percentual & Percentual \\
\hline & Fez até um ano & 35,00 & 39,66 \\
\hline & Fez mais de um até dois anos & 16,49 & 17,66 \\
\hline & Fez mais de dois anos & 15,58 & 17,43 \\
\hline & Nunca fez & 32,93 & 25,25 \\
\hline & Total & 100,00 & 100,00 \\
\hline
\end{tabular}

Fonte: Elaboração própria a partir de dados disponíveis pela PNAD 2008.

Tabela 2. Estatísticas Descritivas das variáveis explicativas.

\begin{tabular}{lrrr}
\hline \multicolumn{1}{c}{ Variáveis } & Média & Mínimo & Máximo \\
\hline Idade & 55,84 & 40 & 108 \\
Branca & 0,54 & 0 & 1 \\
Ensino Fundamental & 0,22 & 0 & 1 \\
Ensino Médio & 0,18 & 0 & 1 \\
Ensino Superior & 0,10 & 0 & 1 \\
Faixa de renda & 3,74 & 0 & 7 \\
Plano de Saúde & 0,31 & 0 & 1 \\
Diagnóstico do câncer & 0,014 & 0 & 1 \\
Autoavaliação da saúde & 2,43 & 1 & 5 \\
Fumante & 0,024 & 0 & 1 \\
Casal sem filhos & 0,19 & 0 & 1 \\
Casal com todos os filhos & 0,02 & 0 & 1 \\
menores de 14 anos & & & \\
Casal com todos os filhos & 0,18 & 0 & 1 \\
maiores de 14 anos & & & \\
Urbana & 0,86 & 0 & 1 \\
Sudeste & 0,47 & 0 & 1 \\
Sul & 0,16 & 0 & 1 \\
Centro-Oeste & 0,07 & 0 & 1 \\
Nordeste & 0,24 & 0 & 1 \\
Norte & 0,07 & 0 & 1 \\
\hline & & & \\
\hline
\end{tabular}

Fonte: Elaboração própria a partir de dados disponíveis pela PNAD 2008.

ou, em oposição, nunca fizeram exames médicos preventivos para diagnóstico de tumor mamário maligno.

Deve-se ressaltar que essas mulheres estão subdivididas numa faixa etária dos 40 a 108 anos (conforme será visto com mais detalhe na Tabela 2). Como já evidenciado, o recorte da amostra em 40 anos de idade se faz pela ascendente incidência do câncer de mama nessa faixa etária.
Para a análise das informações obtidas a partir da amostra, a Tabela 2 apresenta as estatísticas descritivas (especificamente média, mínimo, máximo).

No que se refere aos aspectos socioeconômicos, quanto ao nível educacional, optou-se por considerar dummies para os níveis de escolaridade como: fundamental, médio e superior. Observa-se que a maioria das mulheres brasileiras declararam que têm ensino fundamental, com $22 \%$. Já as mulheres com ensino superior apresentaram $10 \%$ em média. Em relação à faixa de renda domiciliar, uma grande parte declarou que recebe entre um e dois salários mínimos. Quanto ao plano de saúde, que reflete tanto uma característica socioeconômica quanto um aspecto de saúde, nota-se que $31 \%$ das mulheres declararam que têm plano de saúde.

Ainda analisando os aspectos da saúde, 1,4 $\%$ das mulheres declararam que já tiveram câncer, enquanto que a maioria avaliou seu estado de saúde como regular. A Tabela 2 ainda mostra que $2,4 \%$ das mulheres atualmente fumam (ressaltando que o tabagismo está entre os fatores de risco para o desenvolvimento de tumores).

Quanto aos aspectos comportamentais, considerando a composição familiar, constatou-se que $19 \%$ dos casais não têm filhos, $2 \%$ dos casais têm filhos menores de 14 anos e 18\% dos casais têm filhos com idades iguais e/ou maiores de 14 anos. Por último, em termos regionais, verifica-se que $86 \%$ da população feminina reside na região urbana.

\section{Estratégia de estimação: modelo logit ordenado}

Para alcançar as estimativas dos parâmetros (respostas) das variáveis demográficas, socioeconômicas, comportamentais, regionais e de saúde em relação à prevenção do câncer de mama no Brasil, adotou-se um modelo econométrico de escolha discreta, no qual, a variável dependente possui um caráter qualitativo. $\mathrm{Na}$ pesquisa aqui proposta, foram avaliados dois modelos, a saber: i) Estimação dos determinantes da frequência temporal quanto a demanda por exames de mamografia e; ii) Estimação dos preditores da frequência temporal quanto a procura por exames de toque nas mamas.

A apreciação dos modelos e a análise dos dados foram realizadas com uso do pacote estatístico Stata, versão 11.2. Isto posto, o modelo que melhor se ajustou para aferir os resultados foi o de resposta ordenada, o qual se inclui no conjunto 
de modelagens econométricas em que a resposta de uma variável dependente, que pode assumir " $N$ " categorias, é explicada por um número de regressores independentes arbitrariamente escalados $^{15}$. A equação (2) mostra a estrutura do modelo ordinal que pode ser definida como segue:

$$
y^{*}=X^{\prime} \beta+u
$$

A variável dependente (Y) é uma variável qualitativa, da qual são interpretados os efeitos marginais dos dois modelos. De acordo com as informações das entrevistadas, as respostas são distribuídas em caráter ordenado, assim subdivididas, com relação à última vez que fez o exame: 1 até um ano; mais de 1, até 2 anos; mais de 2 e 4 anos, que nunca fez pelo menos um dos exames. Sendo assim, ter-se-á um modelo para aferir a probabilidade 'prevenção ao câncer de mama', tanto avaliado via realização de mamografias, quanto valorado através da realização de exames das mamas, realizados por médicos ou enfermeiros. As variáveis explicativas (vetor X) são de caráter informativo das mulheres, como, por exemplo, se têm filhos menores de 14 anos, como avaliam sua saúde, se residem na região urbana, entre outras.

Considerando que no referido modelo, a variável dependente $\mathrm{y}^{*}$ está substituindo J categorias ordinais, temos:

$$
y=j \quad \text { se } \quad k_{j-1} \leq k_{j} \quad \text { para } 1 \leq j<J
$$

Os limites, $k_{o}$ e $k_{j-1}$, são estimados com $k_{o}=$ $-\infty$ e $k_{j}=+\infty$, de acordo com o pressuposto do modelo. Tem-se, portanto, considerando $j$ categorias que:

$$
\begin{aligned}
& y=0 \quad \text { se } k_{\mathrm{o}}=-\infty \leq y_{1}^{*}<k_{1} \\
& y=1 \quad \text { se } k_{1} \leq y_{1}^{*}<k_{2} \\
& \vdots \\
& y_{j}=J \quad \text { se } \quad k_{\mathrm{j}} \leq y_{1}^{*}=\infty
\end{aligned}
$$

Conforme ressaltam Long e Freese ${ }^{15}$ e Abreu et al. ${ }^{16}$, o modelo padrão ordenado supõe que o coeficiente $(\beta)$ apresente a hipótese de que exista semelhança individual entre as $J$ categorias ordinais da variável dependente e que o valor desses $\beta$, de significância estatística, se mantenha constante ao longo das categorias existentes. Essa proposição é determinada pela Hipótese das Regressões Paralelas.

Contudo, na possibilidade de violação da hipótese do modelo das regressões paralelas, têmse duas possibilidades metodológicas, a saber: $\mathrm{O}$ modelo ordinal generalizado e o modelo ordenado de chances proporcionais parciais.

No modelo generalizado existe à hipótese de que os $\beta$ estimados sejam divergentes para todas as $J$ categorias da variável dependente. Enquanto que o modelo ordenado de chances proporcionais parciais é uma alternativa mais adequada entre as duas já apresentadas, sendo um modelo intermediário entre o padrão e o generalizado.

Neste trabalho, conforme descrição de Abreu et al. ${ }^{16}$ foi considerada, a priori, a adoção dos três modelos, escolhidos em um segundo momento através da realização do teste de Brant ${ }^{17}$, que indicou o modelo de chances proporcionais parciais como o mais apropriado (Tabela 3). Portanto, o modelo ordenado empregado no presente estudo, obteve as estimativas dos efeitos marginais das medidas de frequência do exame mamográfico e exame de mama no Brasil.

No modelo logit, os valores dos coeficientes não são interpretados diretamente, por esse motivo são apresentados apenas os efeitos marginais na seção de resultados. Também cabe destacar que os efeitos marginais devem ser calculados em termos de pontos percentuais e não em termos percentuais. As variações em percentual relacionam-se diretamente ao índice 100, enquanto os pontos percentuais remetem para a diferença em termos absolutos entre quantitativos já em percentagem.

\section{Resultados}

As Tabelas 4 e 5 trazem os resultados da avaliação, utilizando um modelo para dois testes distintos, a saber: i) Identificação do efeito da condição prévia de saúde, status socioeconômico e indicadores comportamentais na determinação da frequência temporal mamográfica realizado pelas mulheres no Brasil; e ii) Identificação do efeito da condição prévia de saúde, status socioeconômico e indicadores comportamentais na determinação da frequência temporal a exames das mamas, realizados por médicos ou enfermeiros, feito pela mulher brasileira, além da verificação da importância de moradia no padrão da prevenção.

O modelo visa explicar quais os determinantes da frequência temporal ao procedimento de mamografia, observando as variáveis selecionadas. O único parâmetro que não se mostrou estatisticamente significativo em todas as categorias foi se o casal possuía filhos menores de 14 anos. Assim, não foram encontradas evidências de que a mulher pertencer a uma família com filhos menores de 14 anos interferia na demanda por prevenção de saúde, avaliada por meio do exame mamográfico computadorizado.

Também verificou que existe um efeito positivo entre a idade e a probabilidade de a mulher 
Tabela 3. Teste de Brant para suposição de regressões paralelas para mamografia e exame da mama.

\begin{tabular}{|c|c|c|}
\hline \multirow{2}{*}{$\frac{\text { Variáveis mamografia }}{\text { Variáveis com indicação para logit ordenado }}$} & \multicolumn{2}{|c|}{ Prob $>\chi^{2}$} \\
\hline & & \\
\hline Centro-Oeste & & 0,5244 \\
\hline Nordeste & & 0,4826 \\
\hline Sul & & 0,2117 \\
\hline Casal sem filhos & & 0,4401 \\
\hline Casal com todos os filhos maiores de 14 anos & & 0,2769 \\
\hline Casal com todos os filhos menores de 14 anos & & 0,2903 \\
\hline Fumante & & 0,0980 \\
\hline Diagnóstico de Câncer & & 0,4104 \\
\hline \multicolumn{3}{|c|}{ Variáveis com indicação para logit ordenado generalizado } \\
\hline Idade & & 0,0000 \\
\hline Idade ao quadrado & & 0,0000 \\
\hline Branca & & 0,02866 \\
\hline Fundamental & & 0,0000 \\
\hline Médio & & 0,0000 \\
\hline Superior & & 0,0000 \\
\hline Plano de saúde & & 0,00356 \\
\hline Avaliação da saúde & & 0,0000 \\
\hline Faixa de renda per capita & & 0,0000 \\
\hline Região Urbana & & 0,0000 \\
\hline Sudeste & & 0,0000 \\
\hline \multirow[t]{2}{*}{ Conjunto das variáveis } & $(16,5188)$ & $=1,23$ \\
\hline & Prob $>\quad F$ & $=0,2353$ \\
\hline \multicolumn{3}{|l|}{ Variáveis exame de mama } \\
\hline \multicolumn{3}{|l|}{ Variáveis com indicação para logit ordenado } \\
\hline Centro-Oeste & & 0,3757 \\
\hline Nordeste & & 0,6042 \\
\hline Casal sem filhos & & 0,1523 \\
\hline Casal com todos os filhos maiores de 14 anos & & 0,0897 \\
\hline Casal com todos os filhos menores de 14 anos & & 0,6126 \\
\hline Fumante & & 0,0672 \\
\hline Diagnóstico de Câncer & & 0,5601 \\
\hline \multicolumn{3}{|c|}{ Variáveis com indicação para logit ordenado generalizado } \\
\hline Idade & & 0,0000 \\
\hline Sul & & 0,0000 \\
\hline Branca & & 0,027 \\
\hline Fundamental & & 0,0000 \\
\hline Médio & & 0,0000 \\
\hline Superior & & 0,0000 \\
\hline Plano de saúde & & 0,0002 \\
\hline Avaliação da saúde & & 0,0000 \\
\hline Faixa de renda per capita & & 0,0000 \\
\hline Região Urbana & & 0,0000 \\
\hline Sudeste & & 0,0000 \\
\hline \multirow[t]{2}{*}{ Conjunto das variáveis } & $(16,5188)$ & $=1,41$ \\
\hline & Prob > F & $=0,1383$ \\
\hline
\end{tabular}

Fonte: Elaboração própria a partir dos resultados fornecidos pelo software utilizado.

ter feito a mamografia há pelo menos um ano. $O$ resultado mostra que o acréscimo de um ano na idade aumentava em 3,14 pontos percentuais (p.p) a probabilidade da mulher ter realizado tal exame, no intervalo mencionado. Outra informação relevante, com respeito à idade, é que exis- 
Tabela 4. Efeitos marginais - Modelo logit ordenado de chances proporcionais parciais - Frequência a prevenção do câncer de mama - (categoria base = Nunca fez mamografia ou nunca fez exame de mama)

\begin{tabular}{|c|c|c|c|c|c|c|}
\hline \multirow[b]{2}{*}{ Variáveis } & \multicolumn{3}{|c|}{ Mamografia } & \multicolumn{3}{|c|}{ Exame da mama } \\
\hline & $\begin{array}{l}\text { Faz até } \\
1 \text { ano }\end{array}$ & $\begin{array}{l}\text { Faz mais de } \\
1 \text { até } 2 \text { anos }\end{array}$ & $\begin{array}{l}\text { Faz mais } \\
\text { de } 2 \text { anos }\end{array}$ & $\begin{array}{l}\text { Faz até } \\
1 \text { ano }\end{array}$ & $\begin{array}{l}\text { Faz mais de } \\
1 \text { até } 2 \text { anos }\end{array}$ & $\begin{array}{l}\text { Faz mais } \\
\text { de } 2 \text { anos }\end{array}$ \\
\hline Avaliação da saúde & $\begin{array}{r}0,045 \\
\end{array}$ & $\begin{array}{r}0,064 \\
(0,012)\end{array}$ & $\begin{array}{r}-0,014 \\
(0,012)\end{array}$ & $\begin{array}{r}0,048 \\
(0,012)\end{array}$ & $\begin{array}{r}0,078 \\
(0,012)\end{array}$ & $\begin{array}{r}0,003 \\
(0,013)\end{array}$ \\
\hline Diagnóstico do câncer & $\begin{array}{r}-0,855 \\
(0,077)\end{array}$ & $\begin{array}{r}-0,855 \\
(0,077)\end{array}$ & $\begin{array}{r}-0,855 \\
(0,077)\end{array}$ & $\begin{array}{r}-0,823 \\
(0,079)\end{array}$ & $\begin{array}{r}-0,823 \\
(0,079)\end{array}$ & $\begin{array}{r}-0,823 \\
(0,079)\end{array}$ \\
\hline Fumante & $\begin{array}{r}0,229 \\
(0,530)\end{array}$ & $\begin{array}{r}0,229 \\
(0,530)\end{array}$ & $\begin{array}{r}0,229 \\
(0,530)\end{array}$ & $\begin{array}{r}0,218 \\
(0,051)\end{array}$ & $\begin{array}{r}0,218 \\
(0,051)\end{array}$ & $\begin{array}{r}0,218 \\
(0,051)\end{array}$ \\
\hline Plano de Saúde & $\begin{array}{r}-0,808 \\
(0,025)\end{array}$ & $\begin{array}{r}-0,844 \\
(0,026)\end{array}$ & $\begin{array}{l}-0,908 \\
(0,031)\end{array}$ & $\begin{array}{r}-0,740 \\
(0,023)\end{array}$ & $\begin{array}{r}-0,799 \\
(0,026)\end{array}$ & $\begin{array}{r}-0,908 \\
(0,037)\end{array}$ \\
\hline Idade & $\begin{array}{r}-0,145 \\
(0,008)\end{array}$ & $\begin{array}{l}-1,555 \\
(0,008)\end{array}$ & $\begin{array}{l}-0,203 \\
(0,008)\end{array}$ & $\begin{array}{r}0,029 \\
(0,0009)\end{array}$ & $\begin{array}{r}0,037 \\
(0,0009)\end{array}$ & $\begin{array}{r}0,0270 \\
(0,0009)\end{array}$ \\
\hline Idade ao quadrado & $\begin{array}{r}0,0014 \\
(0,00006)\end{array}$ & $\begin{array}{r}0,0015 \\
(0,00006)\end{array}$ & $\begin{array}{r}0,0018 \\
(0,00006)\end{array}$ & - & - & - \\
\hline Branca & $\begin{array}{r}-0,077 \\
(0,021)\end{array}$ & $\begin{array}{r}-0,084 \\
(0,021)\end{array}$ & $\begin{array}{r}-0,126 \\
(0,022)\end{array}$ & $\begin{array}{r}-0,769 \\
(0,021)\end{array}$ & $\begin{array}{r}-0,082 \\
(0,021)\end{array}$ & $\begin{array}{r}-0,135 \\
(0,025)\end{array}$ \\
\hline Ensino Fundamental & $\begin{array}{r}-0,186 \\
(0,027)\end{array}$ & $\begin{array}{l}-0,225 \\
(0,025)\end{array}$ & $\begin{array}{l}-0,326 \\
(0,026)\end{array}$ & $\begin{array}{l}-0,119 \\
(0,025)\end{array}$ & $\begin{array}{r}-0,174 \\
(0,025)\end{array}$ & $\begin{array}{r}-0,293 \\
(0,028)\end{array}$ \\
\hline Ensino Médio & $\begin{array}{r}-0,338 \\
(0,030)\end{array}$ & $\begin{array}{r}-0,450 \\
(0,029)\end{array}$ & $\begin{array}{r}-0,581 \\
(0,031)\end{array}$ & $\begin{array}{r}-0,274 \\
(0,279)\end{array}$ & $\begin{array}{c}-0,414 \\
(0,029)\end{array}$ & $\begin{array}{r}-0,608 \\
(0,035)\end{array}$ \\
\hline Ensino Superior & $\begin{array}{r}-0,366 \\
(0,038)\end{array}$ & $\begin{array}{l}-0,450 \\
(0,039)\end{array}$ & $\begin{array}{l}-0,604 \\
(0,049)\end{array}$ & $\begin{array}{l}-0,321 \\
(0,037)\end{array}$ & $\begin{array}{r}-0,450 \\
(0,043)\end{array}$ & $\begin{array}{r}-0,722 \\
(0,063)\end{array}$ \\
\hline Faixa de Renda & $\begin{array}{r}-0,211 \\
(0,009)\end{array}$ & $\begin{array}{r}-0,252 \\
(0,008)\end{array}$ & $\begin{array}{r}-0,288 \\
(0,009)\end{array}$ & $\begin{array}{r}-0,208 \\
(0,009)\end{array}$ & $\begin{array}{r}-0,250 \\
(0,009)\end{array}$ & $\begin{array}{r}-0,294 \\
(0,010)\end{array}$ \\
\hline Casal com filhos maiores de 14 anos & $\begin{array}{r}0,075 \\
(0,022)\end{array}$ & $\begin{array}{r}0,075 \\
(0,022)\end{array}$ & $\begin{array}{r}0,075 \\
(0,022)\end{array}$ & $\begin{array}{r}0,0469 \\
(0,022)\end{array}$ & $\begin{array}{l}0,0469 \\
(0,022)\end{array}$ & $\begin{array}{r}0,0469 \\
(0,022)\end{array}$ \\
\hline Casal com filhos menores de 14 anos & $\begin{array}{r}0,071 \\
(0,063)\end{array}$ & $\begin{array}{r}0,071 \\
(0,063)\end{array}$ & $\begin{array}{r}0,071 \\
(0,063)\end{array}$ & $\begin{array}{r}0,125 \\
(0,063)\end{array}$ & $\begin{array}{r}0,125 \\
(0,063)\end{array}$ & $\begin{array}{r}0,125 \\
(0,063)\end{array}$ \\
\hline Casal sem filhos & $\begin{array}{r}-0,105 \\
(0,022)\end{array}$ & $\begin{array}{r}-0,105 \\
(0,022)\end{array}$ & $\begin{array}{r}-0,105 \\
(0,022)\end{array}$ & $\begin{array}{r}-0,156 \\
(0,021)\end{array}$ & $\begin{array}{r}-0,156 \\
(0,021)\end{array}$ & $\begin{array}{r}-0,156 \\
(0,021)\end{array}$ \\
\hline Urbana & $\begin{array}{l}-0,584 \\
(0,044)\end{array}$ & $\begin{array}{l}-0,583 \\
(0,041)\end{array}$ & $\begin{array}{l}-0,728 \\
(0,040)\end{array}$ & $\begin{array}{l}-0,458 \\
(0,042)\end{array}$ & $\begin{array}{r}-0,497 \\
(0,039)\end{array}$ & $\begin{array}{c}-0,712 \\
(0,042)\end{array}$ \\
\hline Nordeste & $\begin{array}{l}-0,323 \\
(0,045)\end{array}$ & $\begin{array}{l}-0,323 \\
(0,045)\end{array}$ & $\begin{array}{r}-0,323 \\
(0,045)\end{array}$ & $\begin{array}{l}-0,323 \\
(0,042)\end{array}$ & $\begin{array}{r}-0,323 \\
(0,042)\end{array}$ & $\begin{array}{r}-0,323 \\
(0,042)\end{array}$ \\
\hline Centro-oeste & $\begin{array}{l}-0,362 \\
(0,051)\end{array}$ & $\begin{array}{l}-0,362 \\
(0,051)\end{array}$ & $\begin{array}{l}-0,362 \\
(0,051)\end{array}$ & $\begin{array}{r}-0,474 \\
(0,047)\end{array}$ & $\begin{array}{r}-0,474 \\
(0,047)\end{array}$ & $\begin{array}{r}-0,474 \\
(0,047)\end{array}$ \\
\hline Sul & $\begin{array}{l}-0,477 \\
(0,051)\end{array}$ & $\begin{array}{l}-0,477 \\
(0,051)\end{array}$ & $\begin{array}{l}-0,477 \\
(0,051)\end{array}$ & $\begin{array}{l}-0,599 \\
(0,047)\end{array}$ & $\begin{array}{l}-0,573 \\
(0,050)\end{array}$ & $\begin{array}{l}-0,671 \\
(0,054)\end{array}$ \\
\hline Sudeste & $\begin{array}{l}-0,708 \\
(0,047)\end{array}$ & $\begin{array}{l}-0,813 \\
(0,046)\end{array}$ & $\begin{array}{l}-0,965 \\
(0,048)\end{array}$ & $\begin{array}{l}-0,697 \\
(0,042)\end{array}$ & $\begin{array}{l}-0,792 \\
(0,042)\end{array}$ & $\begin{array}{r}-1,006 \\
(0,046)\end{array}$ \\
\hline Constante & $\begin{array}{r}6,301 \\
(0,246)\end{array}$ & $\begin{array}{r}5,977 \\
(0,239)\end{array}$ & $\begin{array}{r}7,512 \\
(0,245)\end{array}$ & $\begin{array}{r}0,821 \\
(0,080)\end{array}$ & $\begin{array}{r}-0,215 \\
(0,080)\end{array}$ & $\begin{array}{r}0,135 \\
(0,081)\end{array}$ \\
\hline Estatística F & 235,99 & & & 252,61 & & \\
\hline P-valor & 0,000 & & & 0,000 & & \\
\hline
\end{tabular}

Fonte: Elaboração própria a partir dos resultados fornecidos pelo software utilizado Nota: Estatística $\mathrm{Z}$ entre parênteses, para $\mathrm{N}=67.722$.

te uma não linearidade nessa variável, ou seja, em um determinado momento da vida o aumento da idade diminui a probabilidade das mulheres realizarem a mamografia. Com isso, observou-se que tudo o mais constante, o padrão de prevenção via idade tende a se reduzir com o tempo, ou seja, aparentemente, as mulheres apresentam uma maior preocupação quando são inseridas no 


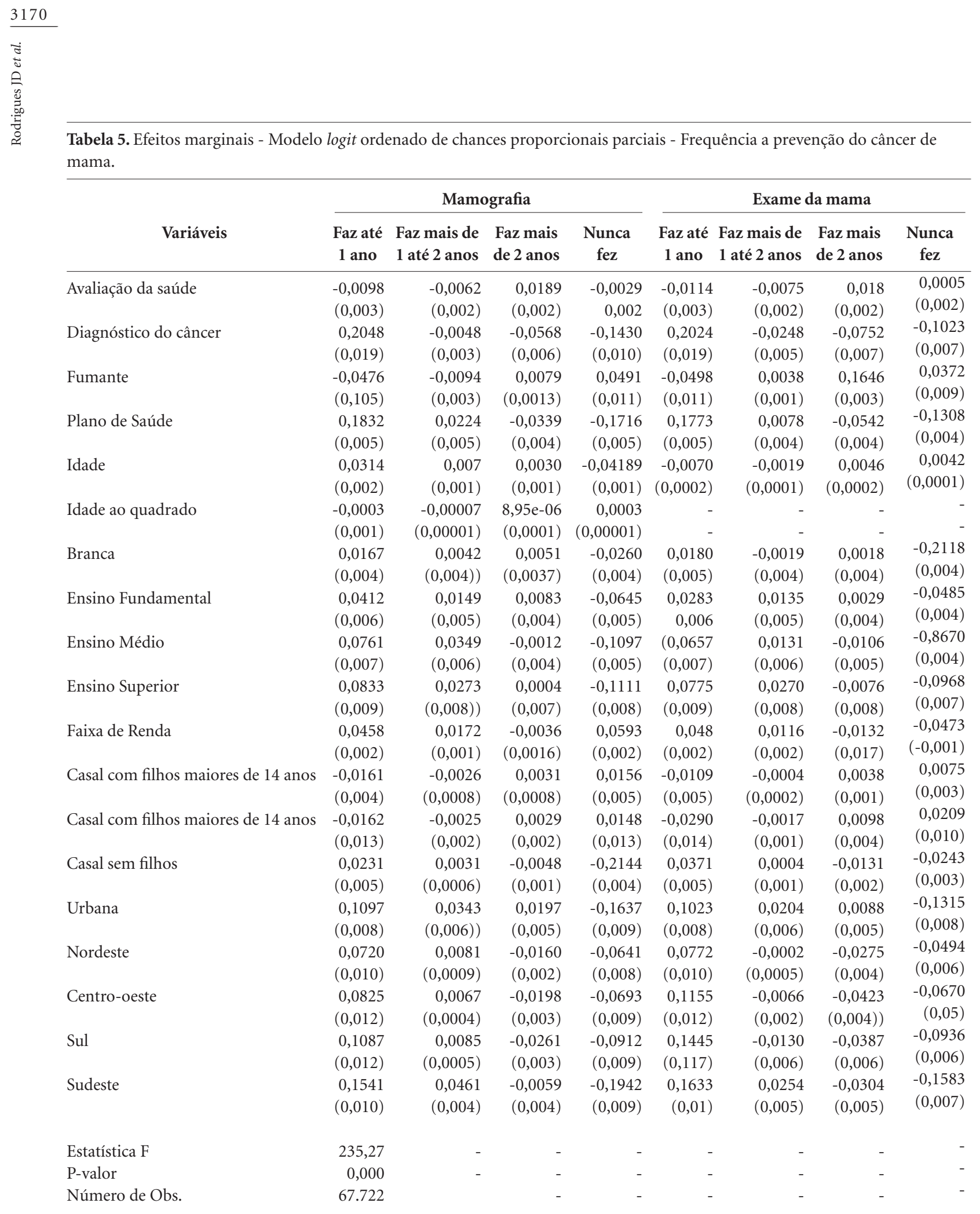

Fonte: Elaboração própria a partir dos resultados fornecidos pelo software utilizado Nota: Erro-padrão entre parênteses, para N=67.511.

grupo de risco mais apontado pela mídia e pelas campanhas de prevenção - faixa pós 40 anos - que, no entanto, com o tempo, reduzem a frequência do exame conforme indicado pela OMS.
Ademais, notou-se que o fato de ser branca aumentou a probabilidade de a mulher fazer o exame mamográfico de maneira mais frequente em 1,67 p.p. Logo, foi possível perceber um 
maior padrão de prevenção entre as autodeclaradas brancas. É importante destacar, apesar de não ser possível inferir, a priori, que esta informação esteja disponível a todas as mulheres de diferentes etnias, que as mulheres brancas são mais propensas a desenvolverem câncer de mama $v i s-a ̀-v i s$ às mulheres de outras cores/raças ${ }^{10}$.

Por outro lado, melhores aspectos socioeconômicos também estão atrelados a uma maior probabilidade da mulher realizar a mamografia ao menos no espaço de um ano. Especificamente, de acordo com o modelo estimado, é possível verificar uma interação positiva da renda e da escolaridade para com a demanda por prevenção da doença, onde um aumento na faixa de rendimentos aumentou em 4,58 p.p a procura pelo exame mamográfico computadorizado, na frequência supracitada.

Dentre as mulheres que possuíam plano de saúde, observou-se um aumento de 18,32 p.p na probabilidade de realização do exame mamográfico no espaço de um ano. Segundo Oliveira e Gonçalves ${ }^{18}$, os indivíduos que possuem plano de saúde são os maiores demandantes de cuidados médicos, resultado este bastante intuitivo, dado que quem tem plano de saúde tende a manter mais regularidade quanto a prevenção à saúde de modo geral.

Ainda se verificou que o fato de a mulher já ter sido diagnosticada com câncer aumentava em 20,48 p.p a probabilidade de ela ter realizado a mamografia todo o ano. Em relação à autoavaliação da saúde da mulher, quanto melhor a avaliação, maior a probabilidade de a mulher realizar a mamografia pelo menos no espaço de um ano. Já entre as mulheres que atualmente fumam, a possibilidade de fazer a mamografia é menor em 4,73 p.p. Infere-se que essas mulheres apresentam uma probabilidade de menor cuidado da saúde, ou seja, a consciência do risco diminui a própria prioridade.

Os resultados apontam também para o fato de que as mulheres que já tiveram câncer são mais propensas a realizarem a mamografia com maior frequência, provavelmente pelo fato de já terem passado por tal problema e também por conhecer melhor a doença e, de tal modo, aumentarem a postura preventiva.

No aspecto comportamental, observou-se que o fato da mulher não ter filhos aumentou em 2,32 p.p a probabilidade da mesma em realizar o exame radiológico das mamas. Verificou-se também que o fato das mulheres terem filhos maiores de 14 anos reduziu a probabilidade em 1,62 p.p da mesma fazer a mamografia.
Além dos indicadores supracitados, a região de moradia também se constituiu em um dos fatores importantes para explicar a frequência da prevenção do câncer de mama, realizado através de procedimentos de mamografia.

Assim, quanto aos aspectos regionais, adotando a região Norte como referência, o Sul e Sudeste são as regiões onde as mulheres mais demandam prevenção, com maior probabilidade, em relação às demais regiões, de 10,87 p.p e 15,41 p.p, respectivamente; enquanto o fato de a mulher residir na área urbana ampliou a probabilidade de fazer o exame mamográfico em 10,97 p.p. Infere-se que as mulheres na região urbana realizam mais exames preventivos do que as residentes em áreas rurais, devido ao acesso aos serviços de saúde que estão concentrados nas cidades.

Isto posto, verificar-se-á agora quais, entre os preditores relacionados, explanam o fato da mulher nunca ter feito um exame de mamografia. É possível observar que apenas a autoavaliação da saúde ou ter filhos menores de 14 anos não interferem no fato da mulher nunca ter realizado o referido exame. Portanto, inicialmente já se observou diferenças em termos dos parâmetros estatisticamente significativos, ao comparar-se as quatro diferentes categorias adotadas pelo modelo. Assim, nota-se a importância da avaliação de um modelo mais parcimonioso, como o modelo Logit, ordenado de chances proporcionais parciais.

Com relação aos demais controles, é possível observar que piores resultados socioeconômicos, residir no Norte do país, atualmente fumar, nunca ter recebido um diagnóstico do câncer de natureza qualquer, ser relativamente mais velha, participar de famílias compostas por filhos maiores de 14 anos ou não ser mãe, desenham o perfil das mulheres que nunca fizeram mamografia.

Analisando alguns dos valores assumidos pelos efeitos marginais, em relação à cor da pele, observa-se que o fato de ser branca diminui a probabilidade de a mulher nunca ter feito a mamografia em 2,61 p.p. Nos aspectos socioeconômicos, entre as mulheres que têm plano de saúde, percebe-se uma diminuição de 17 p.p da probabilidade de nunca realizarem uma mamografia.

Verificando os valores referentes aos aspectos da saúde, percebe-se que o fato da mulher já ter sido diagnosticada com câncer diminui em 14,30 p.p a probabilidade de a mesma nunca fazer o exame mamográfico.

Em síntese, referente aos dados, em especial à parte da população que nunca realizou um exa- 
me mamográfico computadorizado, observa-se um efeito causal contrário, ao comparar-se os resultados observados com o conjunto de mulheres que fizeram o exame há pelo menos um ano: evidencia-se a importância da prevenção.

Como algumas mulheres apresentam restrições à realização de uma mamografia (seja por ressalvas financeiras, culturais ou medo de submeter-se ao exame mamográfico), o presente estudo busca ainda avaliar os determinantes da demanda por prevenção avaliada pela frequência à busca por exames mamográficos de toque realizados por médicos e/ou enfermeiros. Assim, ainda na Tabela 4 é possível verificar os efeitos marginais para o modelo de mensuração da prevenção do câncer de mama, valorada através da realização de exame direto nas mamas, realizado por médico ou enfermeiro (sem o auxílio de equipamento computacional), sendo, portanto, um exame de toque. A atenção de se verificar também a importância dos indicadores da frequência temporal ao procedimento de exames de mamas se faz necessário porque se constitui como outra forma de prevenção, de mais fácil acesso, menos invasiva, do ponto de vista físico, e menos onerosa (dado que mulheres sem plano de saúde, e/ou sem recursos financeiros para providenciarem uma mamografia, podem realizar um exame de toque nas mamas mais facilmente, em postos do PSF's).

Logo, para delinear o modelo em questão, verificando inicialmente para o grupo de mulheres que realizaram o exame há pelo menos um ano, nota-se que todos os parâmetros são estatisticamente significativos. Ademais, de conformidade com o modelo estimado para a avaliação dos determinantes de demanda por prevenção via realização de mamografia, melhores indicadores socioeconômicos, não fumar, ser branca, já ter recebido um diagnóstico de câncer, e não residir no Norte elevam a probabilidade de a mulher realizar o exame de mama em maior frequência. Porém, ao contrário do outro modelo estimado, para o exame de mama foi possível perceber um efeito negativo para as que possuem filhos menores de 14 anos.

Quanto aos aspectos socioeconômicos, assim como foi verificado para a mamografia, a renda domiciliar, a escolaridade e plano de saúde seguem a mesma propensão de aumento das probabilidades de realização do exame de mama. De acordo com este último modelo estimado, um aumento na faixa-renda cresce em 4,83 p.p a demanda por prevenção todo ano. Em relação à escolaridade, a probabilidade de as mulheres com ensino superior fazerem o exame de mama é maior em 7,75 p.p. Já, para o fato de terem plano de saúde, observa-se um aumento de 17,73 p.p da probabilidade de realizar tal procedimento frequentemente (ter feito o exame há pelo menos um ano).

Para os aspectos da saúde, constatou-se um efeito divergente do observado no estudo para a mamografia, ou seja, as mulheres que avaliam de forma positiva a própria saúde são menos propensas à realização de exame de toque nas mamas a menos de um ano (uma melhor avaliação reduz a probabilidade em 1,4 p.p). Por outro lado, ser diagnosticada com câncer aumenta em 420,24 p.p a probabilidade de realização do exame clínico das mamas em até um ano. Dentre as que atualmente fumam, a probabilidade de realização da prevenção reduz-se em 4,98 p.p.

No que se refere ao aspecto comportamental, o modelo mamografia apresentou-se equivalente ao do modelo exame de mama. $\mathrm{O}$ fato da mulher não ter filhos aumenta a probabilidade de realizar exame de mamas em maior frequência. Em contrapartida, as que possuem filhos maiores a 14 anos são menos propensas a uma demanda por prevenção de maneira mais frequente (seja essa prevenção medida pela procura por mamografia ou por exame simples nas mamas). Contudo, o parâmetro casal com filhos menores de 14 anos mostrou-se estatisticamente significativo para o exame de mama, diferentemente do outro modelo estimado, pois para mamografia, não foram encontradas evidências com tal variável que influencie a mulher realizar o exame de mama.

No que diz respeito aos aspectos regionais, também utilizando a região Norte como base, as mulheres das regiões Sudeste e Sul realizam mais frequentemente o exame clínico das mamas, apresentando maior probabilidade em valores de 16,33 p.p e 14,54 p.p, respectivamente. Contudo, o fato da mulher residir na área urbana aumenta em 10,23 p.p a probabilidade de demandar prevenção via exame de mama todo ano.

No grupo intermediário (das mulheres que realizaram o exame clínico das mamas entre um a dois anos e mais de dois anos) não foi possível verificar um padrão entre esses grupos. Contudo, os principais resultados estatisticamente significativos para explicar a frequência da realização do exame de mama nos períodos mencionados foram: diagnosticada com câncer, casal sem filhos, economicamente ativa, plano de saúde. Desta forma, os principais indicadores que explicam o intervalo da prevenção através do exame das mamas por mais de um até dois anos, ou até 
por mais de quatro anos são: socioeconômicos, comportamental e de saúde.

Como já foi mencionada em termos de frequência, a maior parte dos dados concentra-se nos extremos das categorias, isto é, realizaram o exame da mama há pelo menos um ano ou nunca fizeram tal exame. Assim, os resultados do outro extremo - o modelo para as mulheres que nunca realizaram o exame de mama - é perceptível que todos os parâmetros são estatisticamente significativos, ao contrário do modelo mamografia, em que as variáveis economicamente ativas e ter filhos menores de 14 anos não mostram ser parâmetros estatisticamente significativos.

Em relação a idade, os resultados apontam efeito negativo entre a idade e a probabilidade da mulher nunca ter demandado prevenção pelo exame da mama, o resultado mostra que o aumento de um ano na idade amplia em 0,043 p.p a probabilidade da mulher nunca ter feito tal exame. Quanto aos aspectos socioeconômicos, as mulheres com menor nível de escolaridade apresentam menor busca por medidas preventivas via exame das mamas.

Observando os valores no tocante aos aspectos familiares, percebe-se que as mulheres com filhos com idade maior de 14 anos e as que não têm filhos são mais propensas a nunca fazerem o exame das mamas (queda na probabilidade em 0,075 p.p e 0,09 p.p, respectivamente).

Assim como foi verificado para o exame de mamografia, a situação censitária nas regiões $\mathrm{Su}$ deste e Sul aponta redução da probabilidade de a mulher não ter feito o exame clínico das mamas em 15,83 p.p e 9,36 p.p, respectivamente.

\section{Discussão}

De modo geral os resultados encontrados para a demanda por prevenção, por parte da população brasileira das mulheres com mais de 40 anos de idade - considerando grupos desagregados entre as que nunca realizaram qualquer forma de prevenção, as que fizeram o exame há algum tempo e as que realizaram os testes a menos de um ano -, estão associados aos melhores aspectos socioeconômicos, em conformidade com os resultados encontrados por outros autores ${ }^{10-12}$.

Ainda no que se reporta aos aspectos socioeconômicos, os resultados estão condizentes com os encontrados por Politi ${ }^{19}$, que relaciona maior demanda por serviços de saúde com maiores níveis de rendimento, mostrando que quanto maior a renda maior a possibilidade de demandar serviços de saúde.
No entanto, além dos aspectos de caráter educacional e de rendimento, no aspecto comportamental, observa-se o papel da composição familiar na ação de realizar o exame radiológico das mamas. Especificamente, os resultados indicaram que as mulheres sem filhos e as com filhos maiores de 14 anos reduz a probabilidade em 1,62 p.p da mesma fazer a mamografia. Entendese que os efeitos desses resultados influenciam no poder de decisão das mulheres em obter medidas preventivas de saúde. Assim, a questão familiar se mostra importante nos resultados aqui apresentados. Inicialmente, mulheres casadas, com mais de 40 anos, que ainda não têm filhos, estão em uma condição de risco maior do que as demais mulheres (dado que, tudo o mais constante, mulheres com filhos são menos propensas a desenvolverem a doença). Assim, realizar a mamografia com mais frequência pode ser decorrente desta percepção de maior vulnerabilidade. No entanto, por outro lado, é possível considerar também, que a população feminina do Brasil ainda sem filhos possa ser mais previdente com sua saúde, porque ainda desejam exercer a maternidade.

Por outro lado, quanto aos aspectos regionais, destaca-se a importância da região de residência da mulher na frequência à procura por prevenção ao câncer de mama, com destaque para a importância de se residir em regiões urbanas e/ou nas macrorregiões mais desenvolvidas do país. Infere-se que as mulheres na região urbana realizam mais exames preventivos do que as residentes em áreas rurais, devido ao acesso aos serviços de saúde que estão concentrados nas cidades.

Destaca-se também o fato de que a maior parte da população se concentra nos extremos das categorias, isto é, realizaram a mamografia há pelo menos um ano ou nunca realizaram tal exame. Contudo, um fato interessante é que um total de 32,07\% das mulheres no Brasil fez mamografia há mais de um ano. Desagregando os dados, 16,49\% da população feminina fez o exame há mais de um até dois anos, e $15,58 \%$ das brasileiras realizaram o exame há mais de dois anos. Desta parte da população, cabe destacar que não foi possível observar um padrão no que se reporta aos determinantes da demanda por prevenção a saúde, avaliada pela procura por exames mamográficos. No entanto, os principais parâmetros estatisticamente significativos para explicar a frequência à realização de mamografia nos períodos mencionados foram: auto avaliação da saúde, tabagismo, região de residência e composição familiar. Isto é, os principais indicadores que explicam o intervalo da prevenção através da mamografia, por mais 
de um, dois e até por mais de três anos são: regional, saúde e comportamental.

Por fim, referente especificamente à parte da população que nunca realizou um exame mamográfico computadorizado, observou-se um efeito causal contrário, ao comparar-se os resultados aos observados com o conjunto de mulheres que fizeram o exame há pelo menos um ano. Portanto, evidencia-se a importância da prevenção. Esse resultado é corroborado pelo trabalho de Fulton et al. ${ }^{20}$, ao indicar a importância da realização da mamografia, dado que este é um tipo de exame que aponta a existência de tumores não diagnosticados pelo simples toque, e a comunidade médica recomenda o procedimento pelo menos uma vez por ano, em mulheres a partir dos 40 anos

Assim, no bojo desta discussão, observa-se o efeito contrário dos resultados, em relação às que fizeram os exames há pelo menos um ano, $\mathrm{o}$ que manifesta a atenção da prevenção via exame de mama. Deve-se inferir que todas as mulheres devem promover a realização periódica de exame das mamas, para a detecção precoce de qualquer alteração existente em sua mama, para, assim, prevenir e diagnosticar precocemente o câncer.

A verificação agregada dos preditores utilizados na estimação de ambos os modelos, indicam que maiores níveis de renda, educação, maior acesso a informações de saúde, provável preocupação com os entes familiares e residir em áreas mais desenvolvidas favorecem a uma maior postura preventiva, tanto em relação ao exame de mama quanto referente à utilização de mamografia. Entretanto, nota-se que é mais fácil prever a probabilidade da realização da mamografia do que a do exame de mama para o câncer. É possível perceber que o exame de mama implica outro tipo de verificação. Inferimos que, por não envolver necessariamente recursos financeiros e envolver custo de oportunidade (custo do deslocamento físico e tempo de espera alto), as mulheres estão realizando menos demanda por prevenção via exame clínico das mamas.

\section{Considerações finais}

O câncer de mama constitui-se na segunda principal causa de morte entre as mulheres brasileiras, excetuando-se os tumores de pele não mela- noma ${ }^{1}$. Segundo estimativas do INCA, até o fim de 2014, no Brasil, devem surgir 57.120 novos casos dessa neoplasia.

Como a incidência desse tipo de câncer é crescente em mulheres, o presente estudo teve como objetivo geral estudar a inter-relação entre a prevenção do câncer de mama e fatores socioeconômicos, demográficos, comportamentais, regionais e de saúde. Para tanto, utilizou-se as informações do suplemento de saúde da PNAD 2008.

O estudo contemplou apenas mulheres a partir dos 40 anos, devido a maior incidência do câncer nessa faixa etária. A metodologia utilizada para estimar a prevenção no Brasil se deu por meio do modelo Logit ordenado, de chances proporcionais, enquanto a análise e discussão dos resultados foram realizadas pelos efeitos marginais.

Em relação aos resultados, é possível observar no presente estudo que a maioria das mulheres entrevistadas fizeram a mamografia e o exame de mama há pelo menos um ano ou, então, em outro extremo, nunca fizeram tais procedimentos. Não foram encontrados um padrão de comportamento entre as que identificaram sua frequência e as categorias intermediarias seguintes: se havia mais de um até dois anos que tinham feito o exame, ou se havia mais de dois anos que tinham se submetido à avaliação clínica.

Sintetizando de forma geral, os resultados apontam como principais fatores que apresentaram maior prevenção neste estudo foram: melhores condições socioeconômicas, residir nas regiões mais desenvolvidas do país, uma composição familiar que incluía filhos, apresentar uma boa autoavaliação da própria saúde e já ter sido diagnosticada com algum tipo de câncer, explicavam um melhor perfil das mulheres que mais se preveniam contra o câncer de mama, tanto em relação à busca por mamografia, como em relação a uma maior demanda por exame de mama.

Sendo assim, infere-se que é necessária a formulação de políticas públicas que possibilitem atenção integral à saúde da mulher, executando ações favoráveis de promoção da saúde e condições efetivas. Promover a saúde da mulher através da prevenção do câncer de mama significa atuar na proteção a determinados fatores sociais e econômicos, contribuindo para a diminuição da incidência desta doença. 


\section{Colaboradores}

JD Rodrigues, MS Cruz e AN Paixão participaram igualmente de todas as etapas de elaboração do artigo.

\section{Referências}

1. Instituto Nacional do Câncer. [homepage na internet]. Incidência de Câncer no Brasil, Estimativa 2014. [acessado 2014 fev 16]. Disponivel em: http://www.inca.gov. br

2. Organização Mundial da Saúde. Relatório sobre a Saúde no Mundo 2001: saúde mental: nova concepção, nova esperança. [acessado $2014 \mathrm{fev} \mathrm{16]}$. Disponivel em: http://www.who.int/en/

3. Instituto Nacional do Câncer. [homepage na internet]. Programa Nacional de Controle do Câncer de Mama. [acessado $2013 \mathrm{dez}$ 10]. Disponivel em: http://www. inca.gov.br

4. Czeresnia D. Ações de promoção à saúde e prevenção de doenças: o papel da ANS. [Fórum de Saúde Suplementar]. 2003. [acessado $2012 \mathrm{dez} 3$ ]. Disponível em: http://www.ans.gov.br/

5. Asaria P, Chisholm D, Mathers C, Ezzati M, Beaglehole R. Chronic disease prevention: health effects and financial costs of strategies to reduce salt intake and control tobacco use. Lancet 2007; 370(15):2044-2053.

6. Kendall P. Investing in prevention improving health and creating sustainability. Victoria: Office of the Provincial Health Officer; 2010.

7. Boland M, Murphy J. The economic argument for the prevention of ill health at population level. [For Working Group on Public Health Policy Framework] 2012. [acessado 2013 maio 15]. Disponível em: http://www. dohc.ie/consultations/closed/yourhealth/Economics_ of_Prevention.pdf?direct $=1$

8. Ramirez AG, Talavera GA, Villarreal R, Suarez L, Mcalister A, Trapino E, Pérez-Stable E, Marti J. Breast cancer screening in regional Hispanic populations. Health Educ. Res. 2000; 15(5):559-568.

9. Wojcik BE, Spinks MK, Stein CR. Effects of Screening Mammography on the Comparative Survival Rates of African American, White, and Hispanic Beneficiaries of a Comprehensive Health Care System. Br J Cancer 2003; 9(3):175-183.

10. Oliveira EXG, Pinheiro RS, Melo ECP, Carvalho MS. Condicionantes socioeconômicos e geográficos do acesso à mamografia no Brasil, 2003-2008. Cien Saude Colet 2011; 16(9):3649-3664.

11. Matos JC, Pelloso SM, Carvalho MDB. Fatores associados à realização da prevenção secundária do câncer de mama no município de Maringá, Paraná, Brasil. Cad Saude Publica 2011; 27(5):888-898.

12. Oliveira FCR, Kretzmann CK. Determinantes da realização de exame preventivo de mamografia por parte da mulher brasileira. In: Anais do X Encontro Brasileiro de Economia da Saúde; 2011; Porto Alegre.

13. Dailey AB, Kasl SV, Holford TR, Calvocoressi L, Jones BA. Neighborhood-level socioeconomic predictors of nonadherence to mammography screening guidelines. Cancer Epidemiol Biomarkers Prev 2007; 16(11):22932303.

14. Akinyemiju TF. Socio-economic and health access determinants of breast and cervical cancer screening in low-income countries: analysis of the World Health Survey. PLoS ONE 2012; 7(11):1-8. 
15. Long SJ, Fresse J. Regression models for categorical dependent variables using stata. $2^{\text {a }}$ ed. College Station: Stata Press; 2006.

16. Abreu MNS, Siqueira, AL, Caiaffa, WT. Regressão logística ordinal em estudos epidemiológicos. Rev Saude Publica 2009; 43(1):183-194

17. Brant R. Assessing proportionality in the proportional odds model for ordinal logistic regression. Biometrics 1990; 46(4):1171-1178.

18. Oliveira VRD, Gonçalves FDO. Demanda por serviços de saúde: uma análise baseada em dados contáveis. In: Anais do XL Encontro Nacional de Economia(ANPEC); 2012; Porto de Galinhas; 2012. p. 2-20.

19. Politi RB. Desigualdade na utilização de serviços de saúde entre adultos: Uma análise dos fatores de concentração da demanda. Econ. Apl. 2014; 18(1):1-18.

20. Fulton JP, Rakowski W, Jones AC. Determinants of breast cancer screening among inner-city hispanic women in comparison with other inner-city women. Public Health Rep 1995; 110(4):476-482.

Artigo apresentado em 01/09/2014

Aprovado em 16/04/2015

Versão final apresentada em 18/04/2015 